\title{
O impacto da má alimentação infantil à longo prazo na saúde do adulto
}

\author{
Long-term impact of child malnutrition on adult health
}

El impacto a largo plazo de la mala nutrición infantil en la salud de los adultos

Mariana Saracino de Almeida ${ }^{1 *}$, Isabela Costa Linhares ${ }^{1}$, Larissa Sant'Ana Brum ${ }^{1}$, Lucas da Silva Coelho ${ }^{1}$, Manuella da Silva Machado ${ }^{1}$, Patrícia Martins Pinto ${ }^{1}$.

\section{RESUMO}

Objetivo: Analisar o impacto da má alimentação infantil na saúde do indivíduo momentaneamente e à longo prazo, a fim de que se possa agregar conhecimento cientifico sobre o tema abordado e incentivar medidas de promoção à saúde. Métodos: A presente pesquisa trata-se de uma revisão integrativa de literatura embasada em evidências científicas, cuja construção deu-se a partir de: identificação de um tema de interesse, seleção da questão a ser abordada, busca de artigos nas bases de dado digitais, estabelecimento de critérios de inclusão e exclusão, e posterior leitura e discussão dos resultados encontrados. Resultados: A nutrição inf antil, se não realizada e acompanhada de forma adequada, possui um grande impacto negativo na saúde do indivíduo tanto momentaneamente, como à longo prazo, sendo responsável por um maior número de mortes e possuindo uma influência positiva no desenvolvimento de comorbidades e complicações. Considerações finais: Considera-se de suma importância o reconhecimento da inadequação da alimentação infantil com a finalidade de instituir estratégias e políticas de saúde pública para o incentivo e inclusão de práticas nutricionais saudáveis logo nos primeiro anos de vida com o objetivo de formar uma sociedade mais saudável.

Palavras-chave: Nutrição infantil, Desnutrição infantil, Impacto nutrição infantil.

\section{ABSTRACT}

Objective: To analyze the impact of poor infant nutrition on the individual's health momentarily and in the long term, in order to add scientific knowledge on the topic addressed and encourage health promotion measures. Methods: This research is an integrative literature review based on scientific evidence, whose construction was based on: identification of a topic of interest, selection of the question to be addressed, search for articles in digital databases, establishment of inclusion and exclusion criteria, and subsequent reading and discussion of the results found. Results: Child nutrition, if not properly performed and monitored, has a great negative impact on the individual's health both momentarily and in the long term, being responsible for a greater number of deaths and having a positive influence on the development of comorbidities and complications. Final considerations: It is considered of paramount importance to recognize the inadequacy of infant feeding in order to institute public health strategies and policies to encourage and include healthy nutritional practices in the first years of life with the aim of forming a more healthy society.

Key words: Child nutrition, Child malnutrition, Child nutrition impact.

\section{RESUMEN}

Objetivo: Analizar el impacto de la mala nutrición infantil en la salud del individuo de manera momentánea y a largo plazo, con el fin de sumar conocimientos científicos sobre el tema abordado y fomentar medidas de

${ }^{1}$ Universidade de Vassouras (UV), Vassouras - RJ. *E-mail: mariana.saracino@hotmail.com

SUBMETIDO EM: 11/2021

I ACEITO EM: 11/2021

I

PUBLICADO EM: 11/2021 
promoción de la salud. Métodos: Esta investigación es una revisión integradora de la literatura basada en la evidencia científica, cuya construcción se basó en: identificación de un tema de interés, selección de la pregunta a abordar, búsqueda de artículos en bases de datos digitales, establecimiento de criterios de inclusión y exclusión, y posterior lectura y discusión de los resultados encontrados. Resultados: La nutrición infantil, si no se realiza y monitorea adecuadamente, tiene un gran impacto negativo en la salud del individuo tanto de manera momentánea como a largo plazo, siendo responsable de un mayor número de muertes e influyendo positivamente en el desarrollo de comorbilidades y complicaciones. Consideraciones finales: Se considera de suma importancia reconocer la inadecuación de la alimentación infantil para poder instituir estrategias y políticas de salud pública que incentiven e incluyan prácticas nutricionales saludables en los primeros años de vida con el objetivo de formar una sociedad más saludable.

Palabras clave: Nutrición infantil, Desnutrición infantil, Impacto en la nutrición infantil.

\section{INTRODUÇÃO}

No Brasil, a constituição garante o direito à saúde e à alimentação, itens que estão inseridos entre os direitos sociais dos cidadãos. A nutrição apropriada é um requisito básico para a promoção e a proteção da saúde, sendo considerada um fator que determina e condiciona a situação de saúde de indivíduos e da população em geral (JAIME PC, et al., 2018).

O Brasil, assim como outros países em desenvolvimento, está passando por notórias mudanças no processo de saúde/doença. Principalmente nos últimos cinquenta anos, tem sido constatadas alterações na qualidade e na quantidade da alimentação, que, associadas a mudanças no estilo de vida, nas condições socioeconômica e demográficas, causam impactos na saúde da população desses locais (DE SOUZA EB, 2010).

Essas transformações contribuíram com a diminuição da miséria com consequente redução da fome e carência de alimentos, com melhoria ao acesso e diversidade dos mesmos, além da garantia da disponibilidade média de calorias para o consumo, embora ainda existam cerca de 16 milhões de pessoas no Brasil vivendo na pobreza extrema (SANTOS DS, et al., 2019).

Por outro lado, ao mesmo tempo em que se observa a redução constante dos casos de desnutrição, são observadas tendências de crescimento de casos de obesidade em todas as camadas da população, apontando para uma nova conjuntura de problemas relacionados à alimentação e nutrição (COUTINHO JG, et al., 2008).

Assim, vem tornando-se cada vez mais frequente a observação da coexistência de problemas da nutrição infantil tanto pelo déficit como pelo excesso de peso, configurando uma "Transição Epidemiológica Nutricional". No extremo referente ao déficit nutricional, há a desnutrição que causa alterações possivelmente irreversíveis, que impactam no crescimento e no desenvolvimento em longo prazo. Por outro lado, o excesso de peso constitui-se como um grande problema de saúde pública e é classificado como doença crônica não transmissivel (PAIS AB e CARRERA EF, 2009).

As crianças possuem um desenvolvimento acelerado durante os primeiros anos de vida, e a nutrição é de suma importância nessa evolução. Até os seis meses, as necessidades nutricionais de um bebê são atendidas exclusivamente por meio da amamentação. Práticas alimentares inadequadas ou carências nutricionais nessa fase podem causar prejuízos à saúde do lactente. Além disso, quando outros alimentos são adicionados à dieta, inicia-se a construção dos hábitos alimentares do bebê, que poderão permanecer em sua vida adulta (PASSANHA A, et al., 2021).

O comportamento alimentar é complexo: vai desde a compra, a elaboração dos pratos desde, até o ato de se alimentar, sofrendo diferentes influênciass, como ambientais, nutricionais, psicológicos, sociais e culturais. Na infância, o comportamento alimentar é mais passível de interferências externas, principalmente dos familiares, que influenciam diretamente na construção de hábitos das crianças (SILVA GP, et al., 2021). 
Assim, as experiências introdutórias de alimentação da criança com novos sabores, odores e texturas, devem ser valorizadas, pois podem definir seu favoritismo, e com isso, facilitar ou dificultar a adoção de hábitos saudáveis desde o início da vida, além de serem capazes de facilitar o cenário para um futuro nutricional saudável, que desempenhará papel importante na proteção contra comorbidades (VASCONCELOS IN, et al., 2021).

Sendo assim, os primeiros 1000 dias de vida do indivíduo são fundamentais para que as vias imunológicas, endócrinas, metabólicas e outras vias do desenvolvimento humano, se desenvolvam em conjunto com o microbioma para atingir uma relação de equilíbrio. A qualidade e a quantidade da dieta, juntamente com a exposição a toxinas e patógenos, possuem grande repercussão na formação do organismo e sua imunidade (FORGIE AJ, et al., 2020).

Desse modo, este estudo teve como propósito analisar o impacto da má alimentação na infância, podendo ela resultar em um quadro de desnutrição ou de sobrepeso/obesidade, na saúde do indivíduo. Além disso, objetivou-se pesquisar como esse hábito pode levar a consequências imediatas e em longo prazo, desde a infância até a idade adulta e velhice.

\section{MÉTODOS}

Trata-se de uma revisão integrativa de literatura embasada em evidências científicas, que possui o objetivo de analisar a temática em questão, a fim de que se possa agregar conhecimento técnico acerca do tema abordado. Sendo assim, as etapas de construção dessa revisão foram: identificar um tema de interesse e elaborar a questão a ser pesquisada, procurar os artigos nas bases de dados digitais e estabelecer parâmetros de inclusão e exclusão para posterior análise e discussão dos resultados obtidos.

Foram buscados artigos publicados nas bases de dados PubMed, Literatura Latino -americana e do Caribe em Ciências da Saúde (LILACS) e Scientific Electronic Library Online (Scielo). Utilizando os seguintes descritores, separadamente: malnutrition, children malnutrition e malnutrition impact, em inglês, em todas as bases.

$\mathrm{Na}$ base de dados Scielo, as buscas resultaram em um total de 3.682 artigos, no entanto, após utilizar o filtro dos anos 2017 a 2021, restringiu-se para 897 artigos. No PubMed, foram encontrados ao todo 300.093 artigos, contudo, após aplicar o filtro do período específico de 2017 a 2021, reduziu-se para 59.127 artigos, sendo selecionados apenas os artigos que era relevantes para a pesquisa atual. Por fim, na LILACS, foram encontrados no total 7.474 artigos, porém, com a aplicação do filtro que determina o período de 2017 a 2021, encurtou-se para 1071 artigos.

De todas as publicações encontradas nas três bases de dados pesquisadas, foram excluídas as repetidas, além disso, estabeleceu-se parâmetros de inclusão e exclusão, e após a sua aplicação, 8 artigos foram utilizados na elaboração da presente pesquisa. Os critérios de inclusão usados incluem estudos que estão de acordo com a questão norteadora. Já entre os parâmetros de exclusão temos: duplicação entre as bases de dados digitais e artigos que fogem do tema abordado. Além disso, todas as referências dos 8 periódicos selecionados foram analisadas, com o objetivo de selecionar bases confiáveis para a pesquisa. A Figura 1 ilustra os parâmetros usados na seleção das publicações utilizadas. 
Figura 1 - Fluxograma da apuração dos artigos para sintetizar esta revisão.
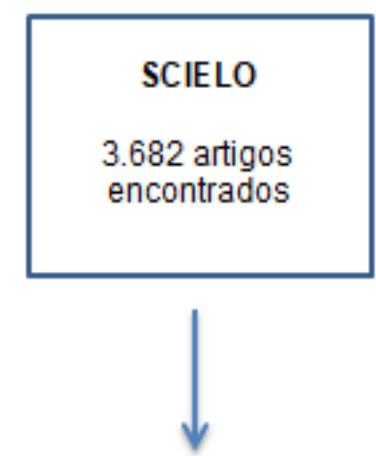
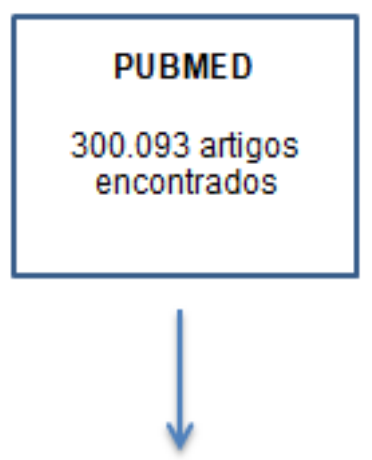

\section{LILAC S}

7.474 artigos encontrados

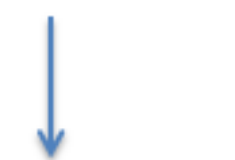

Filtros: artigos publicados entre os anos de 2017 e $2021 \mathrm{em}$ todas as bases.

Parâmetros de inclusão: estudos que estão de acordo com a questão norteadora.

Parâmetros de exclusão: estudos duplicados e que fogem ao tema abordado.
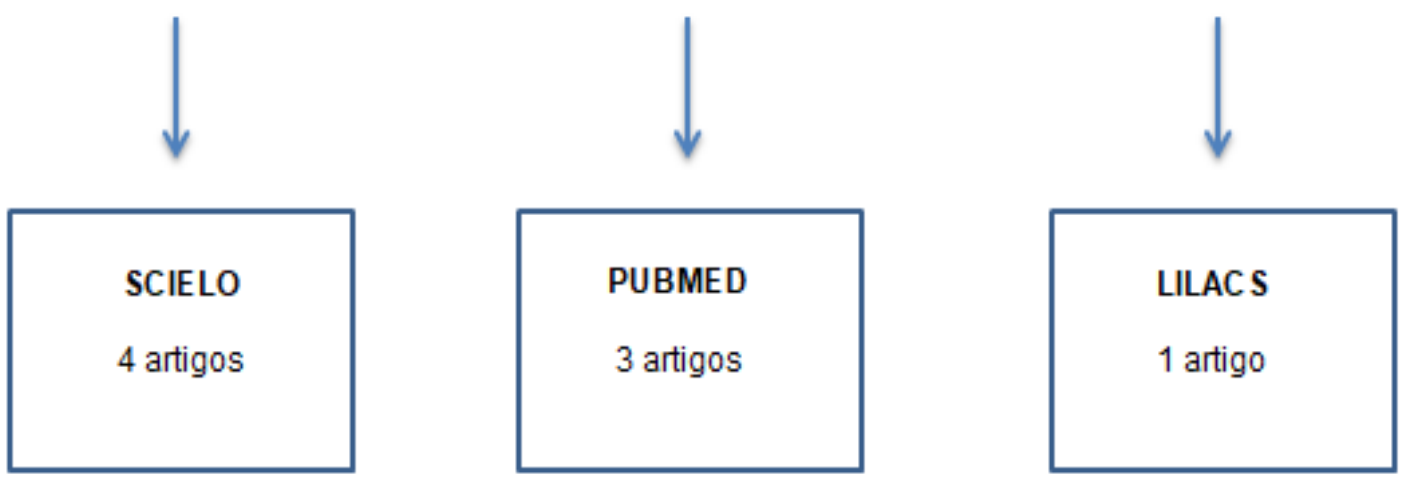

Fonte: ALMEIDA MS, et al., 2021.

\section{RESULTADOS E DISCUSSÃO}

Com base nos 8 artigos estudados, fica claro que, a despeito dos mais diversos perfis epidemiológicos, envolvendo crianças, adultos e idosos, há uma concordância de que a nutrição é de extrema importância nos processos e sistemas do organismo humano, e que os problemas relacionados a ela, seja a obesidad e ou a desnutrição, possuem impacto negativo na saúde dos indivíduos a curto e a longo prazo.

Além disso, fica evidente que a infância é uma etapa de suma importância para o desenvolvimento de habilidades motoras, emocionais, psicológicas e sociais que vão acompanhar o indivíduo pelo resto da vida. Os primeiros anos de vida são determinantes para o progresso infantil e, para que seja pleno, é crucial que haja cuidados principalmente relacionados com a nutrição, como por exemplo, a of erta do Aleitamento Materno Exclusivo (AME) até os seis meses de vida de (PEREIRA TAM, et al., 2021).

Outrossim, os fatores biológicos estudados, como o carente estado nutricional e a desnutrição aguda, tiveram relação significativa com o índice de óbitos (SOUZA NMG, et al., 2020). Ademais, a obesidade também é tratada como um grande problema de saúde pública, uma vez que não só leva doenças agudas na infância, mas acompanha o indivíduo até mais tarde na vida (AKCA SO e BOSTANCI MO, 2017). 
Além disso, em áreas onde os alimentos locais não são suficientes para sup rir às necessidades nutricionais das crianças, a desnutrição é tratada com suplementos alimentares na forma de alimentos misturados, ação que acarretou em uma relação positiva com o desenvolvimento infantil melhorou (OLSEN MF, et al., 2020).

Por isso, a triagem do risco de desnutrição em crianças é preconizada por várias organizações de saúde internacionais, e tem como objetivo identificar indivíduos em perigo de desnutrição para que ações intervencionistas sejam tomadas e, assim, seja evitada a deterioração nutricional (MARDERFELD L, et al., 2019). Isso, em virtude do conhecimento de que a desnutrição pode impactar uma série de tecidos, órgãos, funções do corpo e resultados clínicos, uma vez que está associada a resultados adversos, mortalidade e mal prognóstico em diversas enfermidades (ZIHAO Y, et al. , 2020).

Assim, é importante reconhecer que desenvolver intervenções designadas à melhoria de componentes af etados, encontraram ef eito positivo no desenvolvimento das crianças com algum tipo de necessidade (DA COSTA AGS e NETO JLC, 2019). Portanto, investir na saúde infantil é uma maneira econômica de ter uma população saudável e fornecer evidências que precisam de mais investigação, para elucidação de mecanismos associados (FÉLIX-BELTRÁN L e SEIXAS BV, 2021).

Desse modo, percebe-se que as informações obtida por meio da análise dos artigos escolhidos complementam-se, comprovando que mesmo com perfis epidemiológicos distintos, existe uma concordância entre eles, tornando-os de suma relevâncias para atual conjuntura e para a temática abordada, o que é confirmado por meio das informações encontradas no Quadro 1, para título de análise e exemplificação do que foi exposto. 
Quadro 1 - Resultados obtidos após analise detalhadas dos artigos selecionados.

\begin{tabular}{|c|c|c|c|c|}
\hline Título & Autor & Perfil epidemiológico & Metodologia & Impacto da má nutrição \\
\hline $\begin{array}{l}\text { The impact of anemia } \\
\text { and body mass index } \\
\text { (BMI) on neuromotor } \\
\text { development of } \\
\text { preschool children }\end{array}$ & $\begin{array}{c}\text { AKCA SO e } \\
\text { BOSTANCI MO, } \\
2017\end{array}$ & $\begin{array}{l}916 \text { crianças entre } 5 \text { e } 6 \\
\text { anos matriculadas no } \\
\text { jardim de infância afiliado } \\
\text { à direção de educação de } \\
\text { Çorum entre janeiro e } \\
\text { junho de } 2015 .\end{array}$ & $\begin{array}{l}\text { O Denver Developmental Screening } \\
\text { Test II (DDST II) foi aplicado nas } \\
\text { crianças participantes. Além disso, os } \\
\text { valores de hemoglobina foram } \\
\text { medidos por método não invasivo e o } \\
\text { índice de massa corporal (IMC) foi } \\
\text { calculado. Os dados da pesquisa } \\
\text { foram coletados por meio de } \\
\text { entrevista, questionário, formulário de } \\
\text { avaliação do estado de anemia e } \\
\text { obesidade das crianças e teste de } \\
\text { triagem DDST II. }\end{array}$ & $\begin{array}{l}\text { O estudo constata que a anemia leve possui } \\
\text { relação positiva com o desenvolvimento } \\
\text { neuromotor, enquanto o sobrepeso, a } \\
\text { obesidade e a desnutrição grave, possuem } \\
\text { um impacto negativo. O diagnóstico e a } \\
\text { terapêutica precoces são de suma } \\
\text { importância. Além disso, a pesquisa } \\
\text { constata a relevância do acompanhamento } \\
\text { com programas de rastreamento nutricional } \\
\text { no período pré-escolar e assistência ao } \\
\text { tratamento. }\end{array}$ \\
\hline $\begin{array}{l}\text { Exclusive breastf eeding } \\
\text { and underweight in } \\
\text { children under six } \\
\text { months old monitored in } \\
\text { primary health care in } \\
\text { Brazil. }\end{array}$ & $\begin{array}{c}\text { PEREIRA TAM, } \\
\text { et al., } 2021\end{array}$ & $\begin{array}{c}\text { Foram utilizados dados } \\
\text { de } 4.945 \text { municípios e } \\
167.393 \text { crianças em } \\
\text { relação à antropometria, } \\
\text { e } 1.793 \text { municípios e } \\
66.136 \text { crianças no que } \\
\text { se refere ao consumo } \\
\text { alimentar. }\end{array}$ & $\begin{array}{l}\text { Para a avaliação de consumo } \\
\text { alimentar, foi utilizado um formulário } \\
\text { de marcadores de consumo alimentar, } \\
\text { que tem o objetivo de avaliar os } \\
\text { alimentos consumidos no dia anterior. } \\
\text { Em relação à avaliação do peso, } \\
\text { foram coletadas informações } \\
\text { demográficas (sexo e data de } \\
\text { nascimento) e dados antropométricos } \\
\text { (peso e estatura). }\end{array}$ & $\begin{array}{l}\text { A prevalência de AME no Brasil foi de } \\
56,6 \% \text {, o que a caracteriza dentro da } \\
\text { classificação de um bom indicador, uma vez } \\
\text { que, esse número deveria estar entre } 50 \text { e } \\
\text { 89\%, segundo a classificação da OMS. Em } \\
\text { comparação à Pesquisa Nacional de Saúde, } \\
\text { realizada em } 2013 \text {, evidenciou-se um } \\
\text { aumento, tendo em vista a prevalência de } \\
\text { 45,4\% nesse ano. O estudo demonstra a } \\
\text { necessidade de intensificar as ações e } \\
\text { apresentar novas intervenções de } \\
\text { promoção, proteção e apoio ao AME. }\end{array}$ \\
\hline
\end{tabular}

REAC | Vol. 38 | DOI: https://doi.org/10.25248/REAC.e9272.2021

Página 6 de 12 


\begin{tabular}{|c|c|c|c|c|}
\hline Título & Autor & Perfil epidemiológico & Metodologia & Impacto da má nutrição \\
\hline $\begin{array}{l}\text { Associação do estado } \\
\text { nutricional e os } \\
\text { desfechos clínicos em } \\
\text { cirurgia cardíaca } \\
\text { pediátrica }\end{array}$ & $\begin{array}{l}\text { SOUZA NMG, } \\
\text { et al., } 2020\end{array}$ & $\begin{array}{l}\text { Pacientes com doença } \\
\text { cardíaca congênita com } \\
\text { idade inferior a } 5 \text { anos e } \\
\text { que foram submetidos à } \\
\text { cirurgia cardiovascular no } \\
\text { período de janeiro de } \\
2014 \text { a dezembro de } \\
\text { 2016. Foram analisados } \\
786 \text { prontuários. }\end{array}$ & $\begin{array}{l}\text { A coleta de dados foi feita no período } \\
\text { setembro de } 2017 \text { a abril de } 2018 \text {, por } \\
\text { meio de análise dados em prontuários } \\
\text { físicos. Foi utilizado o Modelo de } \\
\text { Promoção da saúde de Nola J. } \\
\text { Pender como uma base teórica para } \\
\text { analisar os dados deste estudo. } \\
\text { Foram avaliados o estado nutricional } \\
\text { pré-operatório, idade, sexo, peso, } \\
\text { estatural, alta hospitalar, óbitos, } \\
\text { tempo de internação em UTI e tempo } \\
\text { de ventilação mecânica (VM). }\end{array}$ & $\begin{array}{l}\text { O estado nutricional debilitado e a } \\
\text { desnutrição crônica apresentaram relação } \\
\text { significativa com o resultado óbito, } \\
\text { aumentando em } 2,18 \text { e } 2,7 \text { vezes a chance } \\
\text { de morte em crianças submetidas à cirurgia } \\
\text { cardíacas. As crianças com problemas } \\
\text { nutricionais apresentaram maior } \\
\text { necessidade de mais dias utilizando suporte } \\
\text { respiratório invasivo, e consequentemente } \\
\text { mais dias de internação na Unidade de } \\
\text { Terapia Intensiva (UTI). O reconhecimento } \\
\text { precoce de fatores pessoais biológicos } \\
\text { podem ajudar a expandir os resultados } \\
\text { positivos na saúde dessas crianças. }\end{array}$ \\
\hline $\begin{array}{l}\text { Childhood hunger } \\
\text { experiences and chronic } \\
\text { health conditions later in } \\
\text { life among Brazilian } \\
\text { older adults }\end{array}$ & $\begin{array}{c}\text { FÉLIX- } \\
\text { BELTRÁN L e } \\
\text { SEIXAS BV, } \\
2021\end{array}$ & $\begin{array}{l}\text { Ao todo, } 9412 \text { pessoas, } \\
\text { em } 70 \text { municípios de } \\
\text { todas as cinco principais } \\
\text { regiões geográficas do } \\
\text { Brasil fizeram parte da } \\
\text { avaliação inicial. }\end{array}$ & $\begin{array}{l}\text { A avaliação incluiu a coleta de } \\
\text { medidas retrospectivas por meio de } \\
\text { entrevistas, medições físicas e } \\
\text { exames de sangue. Além disso, os } \\
\text { participantes tinha que responder ao } \\
\text { questionamento: "Desde o seu } \\
\text { nascimento até os } 15 \text { anos, você já } \\
\text { teve falta de comida em sua casa e } \\
\text { acabou indo para a cama com } \\
\text { fome?". }\end{array}$ & $\begin{array}{l}\text { Os resultados do estudo mostram que a } \\
\text { associação entre a carência alimentar na } \\
\text { infância foi estatisticamente significativa } \\
\text { para diabetes e osteoporose, mas o mesmo } \\
\text { não foi identificado para hipertensão e } \\
\text { reumatismo. Os indivíduos que relataram } \\
\text { passar fome durante a infância tinham } 20 \% \\
\text { mais chances de desenvolver diabetes } \\
\text { quando adultos e } 38 \% \text { mais chances de } \\
\text { desenvolver osteoporose. Além disso, } \\
\text { afirma a associação entre a baixa estatura } \\
\text { na infância - consequência da fome -e } \\
\text { crescimento do risco de obesidade e } \\
\text { doenças crônico-degenerativas na idade } \\
\text { adulta. }\end{array}$ \\
\hline
\end{tabular}




\begin{tabular}{|c|c|c|c|c|}
\hline Título & Autor & Perfil epidemiológico & Metodologia & Impacto da má nutrição \\
\hline $\begin{array}{l}\text { Association of } \\
\text { malnutrition with all- } \\
\text { cause mortality in the } \\
\text { elderly population: A 6- } \\
\text { year cohort study. }\end{array}$ & $\begin{array}{c}\text { ZIHAO Y, et al., } \\
2020\end{array}$ & $\begin{array}{l}\text { Esse estudo conta com } \\
2.109 \text { participantes de } 60 \\
\text { anos ou mais que foram } \\
\text { inscritos nas ondas de } \\
2009,2011 \text { e } 2015 .\end{array}$ & $\begin{array}{l}\text { Foram coletadas informações } \\
\text { socioeconômicos e antropométricas. } \\
\text { De acordo com os protocolos } \\
\text { padronizados, o índice físico foi } \\
\text { medido por profissionais da saúde } \\
\text { treinados três vezes por participante, } \\
\text { e as médias foram utilizadas nas } \\
\text { análises. Foram coletadas e } \\
\text { estudadas amostras de sangue em } \\
\text { jejum noturno. Os resultados foram } \\
\text { obtidos a partir da relação entre o } \\
\text { peso atual do indivíduo e o peso ideal. }\end{array}$ & $\begin{array}{l}\text { Conclui-se que a desnutrição leve não foi } \\
\text { associada com todas as causas de } \\
\text { mortalidade. No entanto, a desnutrição } \\
\text { severa aumentou o número de óbitos por } \\
\text { todas as causas. Além disso, os pacientes } \\
\text { com valores de albumina sérica baixos } \\
\text { demonstraram internações hospitalares } \\
\text { mais longas, maior risco de hipoproteinemia } \\
\text { e maiores custos de tratamento. Portanto, } \\
\text { análises nutricionais de rotina e assistência } \\
\text { devem ser incluídas no manejo da } \\
\text { população idosa. }\end{array}$ \\
\hline $\begin{array}{l}\text { Validation and impact of } \\
\text { pediatric malnutrition } \\
\text { screening tool in } \\
\text { hospitalised children on } \\
\text { awareness of medical } \\
\text { staff and health-related } \\
\text { outcomes. }\end{array}$ & $\begin{array}{l}\text { MARDERFELD } \\
\text { L, et al., } 2019\end{array}$ & $\begin{array}{c}\text { Crianças entre } 1 \text { e } 17 \\
\text { anos, internadas por mais } \\
\text { de } 24 \text { horas. A análise } \\
\text { final incluiu } 60 \text { crianças } \\
\text { ( } 30 \text { de cada } \\
\text { departamento). }\end{array}$ & $\begin{array}{l}\text { Foram utilizados procedimentos } \\
\text { padronizados para medidas de peso e } \\
\text { comprimento / altura e esses dados } \\
\text { foram utilizados para a avaliação } \\
\text { nutricional. As medições foram } \\
\text { colocadas em gráficos de referência } \\
\text { de crescimento. No final do estudo, foi } \\
\text { realizada regressão logística para } \\
\text { avaliar o impacto da triagem nos } \\
\text { desfechos na alta. }\end{array}$ & $\begin{array}{l}\text { Concluiu-se, que não há repercussão } \\
\text { significativa do conhecimento do estado } \\
\text { nutricional dos pacientes nos resultados } \\
\text { relacionados à sua saúde. Porém, a } \\
\text { desnutrição em crianças está relacionada a } \\
\text { maiores taxas de infecção, complicação, má } \\
\text { cicatrização, tempo prolongado de } \\
\text { permanência, maior taxa de mortalidade e } \\
\text { redução da qualidade de vida. E por isso, a } \\
\text { triagem do risco de desnutrição em crianças } \\
\text { é preconizada por uma série de } \\
\text { organizações. }\end{array}$ \\
\hline
\end{tabular}

REAC | Vol. 38 | DOI: https://doi.org/10.25248/REAC.e9272.2021

Página 8 de 12 


\begin{tabular}{|c|c|c|c|c|}
\hline Título & Autor & Perfil epidemiológico & Metodologia & Impacto da má nutrição \\
\hline $\begin{array}{l}\text { Impact of food } \\
\text { supplements on early } \\
\text { child development in } \\
\text { children with moderate } \\
\text { acute malnutrition: A } \\
\text { randomised } 2 \times 2 \times 3 \\
\text { factorial trial in Burkina } \\
\text { Faso. }\end{array}$ & $\begin{array}{l}\text { OLSEN MF, et } \\
\text { al., } 2020\end{array}$ & $\begin{array}{c}\text { Das } 1.609 \text { crianças } \\
\text { matriculadas, } 54,7 \% \\
\text { eram do sexo feminino e } \\
\text { a idade média foi de } 11,3 \\
\text { meses. } 0 \\
\text { acompanhamento de } 12 \\
\text { semanas foi concluído } \\
\text { em } 1,548(96,2 \%) \text { e } \\
\text { acompanhamento de } 24 \\
\text { semanas foi concluído } \\
\text { por } 1.503(93,4 \%) ;\end{array}$ & $\begin{array}{l}\text { Ensaio fatorial } 2 \times 2 \times 3 \text { randomizado } \\
\text { para avaliar a eficiência da } \\
\text { suplementação nutricional. O estudo } \\
\text { foi totalmente cego com relação à } \\
\text { qualidade da soja e teor de leite, } \\
\text { enquanto os participantes do estudo } \\
\text { não foram cegos com relação à } \\
\text { matriz. }\end{array}$ & $\begin{array}{l}\text { A análise mostra que o desenvolvimento } \\
\text { das crianças participantes melhorou com a } \\
\text { suplementação para o tratamento da } \\
\text { desnutrição aguda moderada (MAM). Os } \\
\text { suplementos a base de lipídeos (LNS) } \\
\text { tiveram maior melhora do que o suplemento } \\
\text { de milho-soja (CSB). Os escores z seguiram } \\
\text { aumentando, mostrando um ef eito } \\
\text { sustentado. }\end{array}$ \\
\hline $\begin{array}{l}\text { Desenvolvimento da } \\
\text { motricidade fina em } \\
\text { crianças com } \\
\text { desnutrição crônica }\end{array}$ & $\begin{array}{c}\text { COSTA AGS e } \\
\text { NETO JLC, } \\
2019\end{array}$ & $\begin{array}{l}\text { A população analisada do } \\
\text { estudo foi formada por } \\
\text { crianças com idades } \\
\text { entre } 24 \text { e } 48 \text { meses, } \\
\text { divididas em casos e } \\
\text { controles para o estudo. } \\
\text { Participaram da pesquisa } \\
23 \text { crianças no total, } \\
\text { sendo } 11 \text { crianças no } \\
\text { CREN e } 12 \text { crianças da } \\
\text { creche municipal. }\end{array}$ & $\begin{array}{c}\text { A pesquisa foi feita no Centro de } \\
\text { Recuperação e Educação Nutricional } \\
\text { (CREN), em Maceió, assim como em } \\
\text { uma creche municipal. Os } \\
\text { instrumentos utilizados foram: escala } \\
\text { de desenvolvimento motor (EDM) e } \\
\text { avaliação antropométrica. Aplicou-se } \\
\text { a bateria de testes de Rosa Neto com } \\
\text { o objetivo de avaliar o } \\
\text { desenvolvimento motor. }\end{array}$ & $\begin{array}{c}\text { Os dados obtidos na avaliação motora } \\
\text { foram inferiores em crianças com o quadro } \\
\text { de desnutrição crônica com relação ao de } \\
\text { crianças eutróficas, em outras palavras, os } \\
\text { dados mostram que a desnutrição pode } \\
\text { af etar direta e/ou indiretamente o } \\
\text { crescimento e o desenvolvimento de uma } \\
\text { criança. }\end{array}$ \\
\hline
\end{tabular}

Fonte: ALMEIDA MS, et al., 2021. 
A dificuldade nutricional na infância é um fator que possui um impacto negativo nos indivíduos que vai além de seu estado de saúde momentâneo. Um número crescente de estudos tem mostrado que uma pluralidade de circunstâncias de desvantagens tem ef eitos de grande gravidade e em longo prazo na saúde de indivíduos. Pesquisas demonstram, por exemplo, uma associação entre a fome na infância e duas doenças crônicas encontradas em indivíduos na vida adulta: diabetes e osteoporose (FÉLIX-BELTRÁN L e SEIXAS BV, 2021).

A despeito de a obesidade ser considerada um dos grandes problemas de saúde pública nos dias de hoje, a desnutrição requer uma atenção especial, uma vez que possui influência direta sobre a condição de saúde do indivíduo. Ainda na infância, possui relação com o desenvolvimento cognitivo e neuropsicomotor. Assim, crianças que passaram por períodos de privação nutricional, se tornam mais suscetíveis às sequelas pós doença (COSTA AGS e NETO JLC, 2019).

Desse modo, a desnutrição é responsável por $30 \%$ das mortes de crianças menores de 5 anos, atingindo a marca de $50 \%$ nos países em desenvolvimento (SILVEIRA VNC, et al., 2020). No Brasil, os números da obesidade também são preocupantes, uma vez que dados do Instituto Brasileiro de Geografia e Estatística (IBGE) mostram que $51,4 \%$ dos meninos e $43,8 \%$ das meninas com idade entre 5 a 9 anos apresentavam sobrepeso ou obesidade, fato que está associada com a hipertensão arterial, diabetes mellitus, dislipidemia e aumento da morbimortalidade cardiovascular na idade adulta (ARANHA LN e OLIVEIRA GMM, 2020).

Além disso, trabalhos demonstram que as adversidades na nutrição de crianças, quando ocorrem em estágios críticos do crescimento e desenvolvimento encefálico, podem impactar gravemente na cognição, uma vez que o cérebro não recebe os nutrientes necessários para seu desenvolvimento. As consequências do déficit de macro e micronutrientes na infância podem não ser reversíveis, e permanecem afetando atividades cognitivas e qualidade de vida até a idade adulta (ZUANETTI PA, et al., 2019).

Assim, a desnutrição e a obesidade são os dois extremos da má alimentação das crianças, e ambos possuem impacto negativo nos processos bioquímicos, ação celular, crescimento, desenvolvimento mental e comportamental, sistema imunológico, capacidade física e diversos outros sistemas do organismo (AKCA SO e BOSTANCI MO, 2017). Por isso, onde os alimentos encontrados não são suficientes para suprir às demandas nutricionais, a suplementação é uma boa opção a fim de evitar consequências negativas no desenvolvimento inf antil (OLSEN MF, et al., 2020).

A nutrição infantil tem inicio ainda durante a gravidez, uma vez que a alimentação materna possui influência direta no desenvolvimento do feto. Hábitos saudáveis durante a gestação, como dieta equeilibrada e atividade física regular possuem um papel de fatores protetores contra eventos adversos como macrossomia, diabetes gestacional, obesidade futura materno-inf antil, entre outros (MALTA MB, et al., 2021).

A alimentação infantil é introduzida logo ao nascimento com o aleitamento materno, que é um ato de promoção à saúde indicado de maneira exclusiva durante os primeiros seis meses de vida, devendo ser iniciada, preferencialmente, ainda na primeira hora de vida. É um alimento inato às mães e que pro porciona grandes benefícios ao lactente, uma vez que dispõe de todos os nutrientes necessários para o seu desenvolvimento (PINTO SL, et al., 2021).

Sendo assim, crianças que são amamentadas adequadamente e pelo tempo recomendado possuem menores índices de morbidade e mortalidade por diarreias, infecções do aparelho respiratório e auditivo, apresentam proteção contra a obesidade e diabetes ao longo da vida. Além disso, há vantagens também para as lactantes, uma vez que o ato de amamentar ajuda a precaver o câncer de mama e ovário e diminuir as chances de desenvolver diabetes (PEREIRA TAM, et al., 2021).

Além dos impactos imediatos e daqueles que acompanham o indivíduo pelo resto da vida citados, a má nutrição possui repercussão em situações de urgência e emergência, como cirurgias. As maiores probabilidades de letalidade pós-operatória, entre aqueles com déficit nutricional e desnutrição crônica foi consistente, uma vez que estudos mostram uma relação entre essa carência e o crescimento do número de mortes no período pós-operatório em crianças expostas à cirurgia cardíaca, por exemplo (SOUZA NMG, et al., 2020). 
Ademais, seguindo as orientações de diversas organizações internacionais, em alguns países passou a ser mandatório realizar a triagem nutricional em crianças no momento da admissão hospitalar, já que a má nutrição pediátrica possui relação direta com maior número de infecção, maior risco de complicações, problemas de cicatrização, tempo aumentado de permanência hospitalar, maior taxa de mortalidade e piora da qualidade de vida (MARDERFELD L, et al., 2019).

Assim, a associação entre o déficit nutricional na infância e o aumento do número de mortes e comorbidades ao longo da vida pode ser compreendida como uma associação de diversos fatores e mecanismos. Diferentes sistemas celulares são influenciados pela nutrição do indivíduo no que tange o gasto de energia, metabolismo das células, sistema imunológico e funcionamento do organismo como um todo. Nesse sentido, a má nutrição pode impactar negativamente as células T, limitando a resposta inflamatória ou a formação de citocinas e as respostas celulares (ZIHAO Y, et al. , 2020).

Portanto, a desnutrição está associada a um quadro de imunossupressão. Pacientes imunodeficientes apresentam um risco aumentado de cursar com infecção e complicações, o que pode levar à morte. A desnutrição possui um grande impacto em quase todos os tecidos e órgãos organismo humano e, progressivamente, leva à degeneração física e mental. Além disso, pode, ainda, ser um fator de piora para as doenças subjacentes, aumentando a sua mortalidade e morbidade (ZIHAO Y, et al. , 2020).

Logo, é primordial o reconhecimento da inadequação da alimentação infantil com a finalidade de instituir estratégias e políticas de saúde pública para o incentivo e inclusão de práticas nutricionais saudáveis logo nos primeiros anos de vida (PEDRAZA DF e SANTOS EES, 2021). Por isso, investir na segurança nutricional das crianças é uma das melhores maneiras de construir uma sociedade mais saudável no futuro (FÉLIXBELTRÁN L e SEIXAS BV, 2021).

\section{CONSIDERAÇÕES FINAIS}

Em suma, é importante salientar que a nutrição infantil, se não realizada e acompanhada de forma adequada, possui um grande impacto negativo na saúde do indivíduo tanto momentaneamente, como à longo prazo. Esse estudo tem como objetivo levar os profissionais da área da saúde a refletir criticamente a respeito da relevância da nutrição pediátrica no que tange a sua quantidade e, principalmente, qualidade, uma vez que ela interfere de forma direta no processo saúde/doença. Além disso, observou-se uma carência de pesquisas recentes sobre o tema discutido, portanto é mister estimular a realização de novos estudos acerca sobre o tema para que então seja melhor analisada na comunidade científica.

\section{REFERÊNCIAS}

1. AKCA SO, BOSTANCI MO. The impact of anemia and body mass index (BMI) on neuromotor development of preschool children. Revista da Associação Médica Brasileira, 2017;63(9): 779-786.

2. ARANHA LN, OLIVEIRA GMM. Circunferência da Cintura, uma Medida Simples para a Obesidade Infantil? Arquivos Brasileiros de Cardiologia, 2020;114(3):538-539.

3. COSTA AGDS, CAVALCANTE JP. Desenvolvimento da motricidade fina em crianças com desnutrição crônica. Cadernos Brasileiros de Terapia Ocupacional, 2019;27(1):54-60.

4. COUTINHO JG, et al. A desnutrição e obesidade no Brasil: o enfrentamento com base na agenda única da nutrição. Cadernos de Saúde Pública, 2008;24(2): 332-340.

5. FÉLIX-BELTRÁN L, SEIXAS BV. Childhood hunger experiences and chronic health conditions later in life among Brazilian older adults. Revista Panamericana de Salud Pública, 2021;45: 1.

6. FORGIE AJ, et al. The impact of maternal and early life malnutrition on health: a diet-microbe perspective. BMC Medicine, 2020;18(1): 135.

7. JAIME PC, et al. Um olhar sobre a agenda de alimentação e nutrição nos trinta anos do Sistema Único de Saúde. Ciência \& Saúde Coletiva, 2018;23(6):1829-1836.

8. MALTA MB, et al. Effectiveness of an intervention focusing on diet and walking during pregnancy in the primary health care service. Cadernos de Saúde Pública, 2021;37(5).

9. MARDERFELD L, et al. Validation and impact of paediatric malnutrition screening tool in hospitalised children on awareness of medical staff and health-related outcomes. Nutrition \& Dietetics, 2019; 76(5):574-579. 
10. OLSEN MF, et al. Impact of food supplements on early child development in children with moderate acute malnutrition: A randomised $2 \times 2$ × 3 factorial trial in Burkina Faso. PLOS Medicine, 2020;17(12): e1003442.

11. PAIS AB, CARRERA EF. Necessidades básicas insatisfeitas, desnutrição infantil e risco de doenças na vida. Revista Paulista de Pediatria, 2009;27(2): 127-132.

12. PASSANHA A, et al. Determinants of fruits, vegetables, and ultra-processed foods consumption among infants. Ciência \& Saúde Coletiva, 2021;26(1): 209-220.

13. PEDRAZA DF, SANTOS EES. Marcadores de consumo alimentar e contexto social de crianças menores de 5 anos de idade. Cadernos Saúde Coletiva, 2021;29(2): 163-178.

14. PEREIRA TADM, et al. Exclusive breastfeeding and underweight in children under six months old monitored in primary health care in brazil, 2017. Revista Paulista de Pediatria, 2021; 39.

15. PINTO SB, et al. Evaluation of breastfeeding self-effectiveness and its associated factors in puerperal women assisted at a public health system in Brazil. Revista Brasileira de Saúde Materno Infantil, 2021;21 (1): 89-96.

16. SANTOS DS, et al. Transição nutricional na adolescência:uma abordagem dos últimos 10 anos. Revista Eletrônica Acervo Saúde, 2019;20(20): e477.

17. SILVA GP, et al. Family influence on the nutritional status and eating habits of six to nine year-old children. Revista de Nutrição, 2021;34.

18. SILVEIRA VNDC, et al. Desnutrição e fatores associados em crianças quilombolas menores de 60 meses em dois municípios do estado do Maranhão, Brasil. Ciência \& Saúde Coletiva, 2020;25(7): 2583-2594.

19. SOUZA EB. Transição nutricional no Brasil: análise dos principais fatores. Cadernos UniFOA, 2017;5(13):49.

20. SOUZA NMG, et al. Associação do estado nutricional e os desfechos clínicos em cirurgia cardíaca pediátrica. Acta Paulista de Enfermagem, 2020;33.

21. VASCONCELOS IN, et al. Breastfeeding and infant feeding guidelines: dietary patterns and potential effects on the health and nutrition of children under two years. Revista Brasileira de Saúde Materno Infantil, 2021; 21 (2):419-428.

22. YU Z, et al. Association of malnutrition with all-cause mortality in the elderly population: a 6-year cohort study. Nutrition, Metabolism and Cardiovascular Diseases, 2021;31(1):52-59.

23. ZUANETTI PA, et al. Early undernutrition as a cause of changes in phonological processing skills. Revista CEFAC, 2019;21(3). 